\title{
Helium white dwarf cooling in PSR J0751+1807 and PSR $\mathrm{J} 1012+5307$
}

\author{
Ene Ergma, J.Antipova \\ Department of Physics, University of Tartu, Ülikooli 18, EE2400 Tartu, \\ Estonia
}

and M. J. Sarna

N. Copernicus Astronomical Center, Polish Academy of Sciences, ul. Bartycka 18, 00-716 Warsaw, Poland

\section{Introduction}

It is accepted that formation of a binary millisecond (or recycled) pulsar with a low-mass companion may be explained as the end-point of close binary evolution in which an old pulsar is spun-up by accretion from the secondary (Alpar et al., 1982). After detachment from the Roche lobe, the pulsar spin period starts to change due to magneto-dipole radiation and the white dwarf begins to cool down. In this paper we shall discuss the cooling history of helium core lowmass white dwarfs in the short orbital period millisecond binary pulsars PSR J0751+1807 and PSR J1012+5307 (Ergma, Sarna, \& Antipova 1999).

\section{Observational data and evolutionary considerations}

In Table 1 we present main observational data for PSRJ0751+1807 and PSR J1012+5307. To produce short orbital period systems with a low-mass helium white dwarf and a millisecond pulsar, it is necessary that the low-mass secondary (red giant) fills its Roche lobe below the so called bifurcation period and above the boundary period (Ergma \& Sarna 1996; Ergma, Sarna, \& Antipova 1998). Ergma et al. (1998) and recent calculations by Sarna, Ergma, \& Antipova (1999) have shown that after detachment of the donor star from its Roche lobe, a rather thick hydrogen layer is left on the top of the helium core. This thick hydrogen layer will greatly influence the cooling history of the very low-mass helium white dwarf $\left(<0.25 M_{\odot}\right)$. Alberts et al. (1996) were the first to argue that since white dwarfs with $M_{w d}<0.20 \quad M_{\odot}$ do not show thermal flashes (as found by Webbink 1975), then the amount of hydrogen in the envelope remains high and results in a much longer phase of significant hydrogen burning. Driebe et al. (1998) and our recent calculations (Sarna et al. 1999) confirm this result. This removes the discrepancy between the cooling and spin-down ages for the millisecond binary systems PSR J1012+5307 (Alberts et al. 1996; Sarna, Antipova, \& Muslimov 1998; Driebe et al. 1998). But now a question arises for the PSR J0751+1807. The evolutionary history for both pulsar systems is very similar, as argued by Ergma \& Sarna (1996). This means that in PSR J0751+1807 the helium white dwarf must also be in a long, stable hydrogen burning phase, which leads to considerably long cooling times and a hot white dwarf. However, observations 
Table 1. Systems parameters

\begin{tabular}{llcccr}
$\begin{array}{llc}\text { system } \\
{[P S R]}\end{array}$ & $P$ & $\dot{P}$ & $P / 2 \dot{P}$ & $t_{\text {cool }} / m_{v} *$ & $M_{w d}$ \\
& {$[m s]$} & {$\left[s s^{-1} / 10^{-20}\right]$} & {$[G y r]$} & {$[G y r / m a g]$} & {$\left[M_{\odot}\right]$} \\
\hline $\mathrm{J} 0751+1807^{3,4}$ & 3.5 & 0.8 & $\sim 8$ & $>23.5^{*}$ & 0.1 \\
$\mathrm{~J} 1012+5307^{1,2,6}$ & 5.256 & 1.46 & $\sim 7$ & 0.3 & $0.16 \pm 0.02$ \\
\hline \hline
\end{tabular}

${ }^{1}$ Callanan, Garnavich, \& Koester $1998 ;{ }^{2}$ Lorimer et al. $1995 ;{ }^{3}$ Lundgren, Zepka, \& Cordes 1995; ${ }^{4}$ Lundgren et al. 1996; ${ }^{5}$ Nicastro et al.1995; ${ }^{6}$ van Kerkwijk, Bergeron, \& Kulkarni 1996.

show that the white dwarf in this system is undetectable and really very old. To solve this discrepancy, we propose that irradiation of the donor star by the millisecond pulsar PSR J0751+1807 may cause from the secondary additional mass loss after detachment from the Roche lobe.

\section{Simple cooling model for the white dwarf in PSR J0751+1807}

To illustrate how the irradiative mass loss will influence the cooling evolution of the pre-white dwarf (after detachment from Roche lobe) we computed the evolutionary sequence for a system of $1.5+1.4 M_{\odot}$, with an initial orbital period 1.07 days and $Z=0.02$. After detachment from the Roche lobe, irradiative mass loss is defined by the simple van den Heuvel \& van Paradijs (1988) model

$$
\dot{M}_{w i n d}=f \frac{R_{w d}}{G M_{w d}}\left(\frac{R_{w d}}{2 a}\right)^{2} \frac{2 R_{n s}^{6}}{3 c^{3}} B^{2}\left(\frac{2 \pi}{P}\right)^{4}
$$

where $B$ and $R_{n s}$ are the magnetic field strength and radius of the neutron star respectively, $R_{w d}$ is radius of the white dwarf, $f$ is efficiency factor (from 0 to 1) and $a$ is the separation between the stars.

Figure 1 shows the evolution of the visual magnitude $\left(m_{V}\right)$ of the progenitor of the white dwarf during the cooling phase, with wind mass loss included. The decrease of the mass of the hydrogen envelope leads to a significant decrease of the hydrogen shell burning efficiency. Therefore, if compared to the evolution of a white dwarf with stationary hydrogen shell burning, the cooling time is decreased. For example, when $\log \mathrm{L} / L_{\odot}=-2.0$, the cooling age without mass loss is $8.4 \times 10^{9} \mathrm{yrs}$, with mass loss $(\mathrm{f}=0.1)$ is $5.07 \times 10^{9} \mathrm{yrs}$, and with a very efficient wind $(\mathrm{f}=0.5)$ is $2.17 \times 10^{9}$ yrs. Figure 1 gives the limit for acceptable $m_{V}$ from theoretical cooling tracks. We can exclude normal cooling (with shell hydrogen burning) due to too high a $m_{V}$ (see Table 1). Two other cases of cooling 


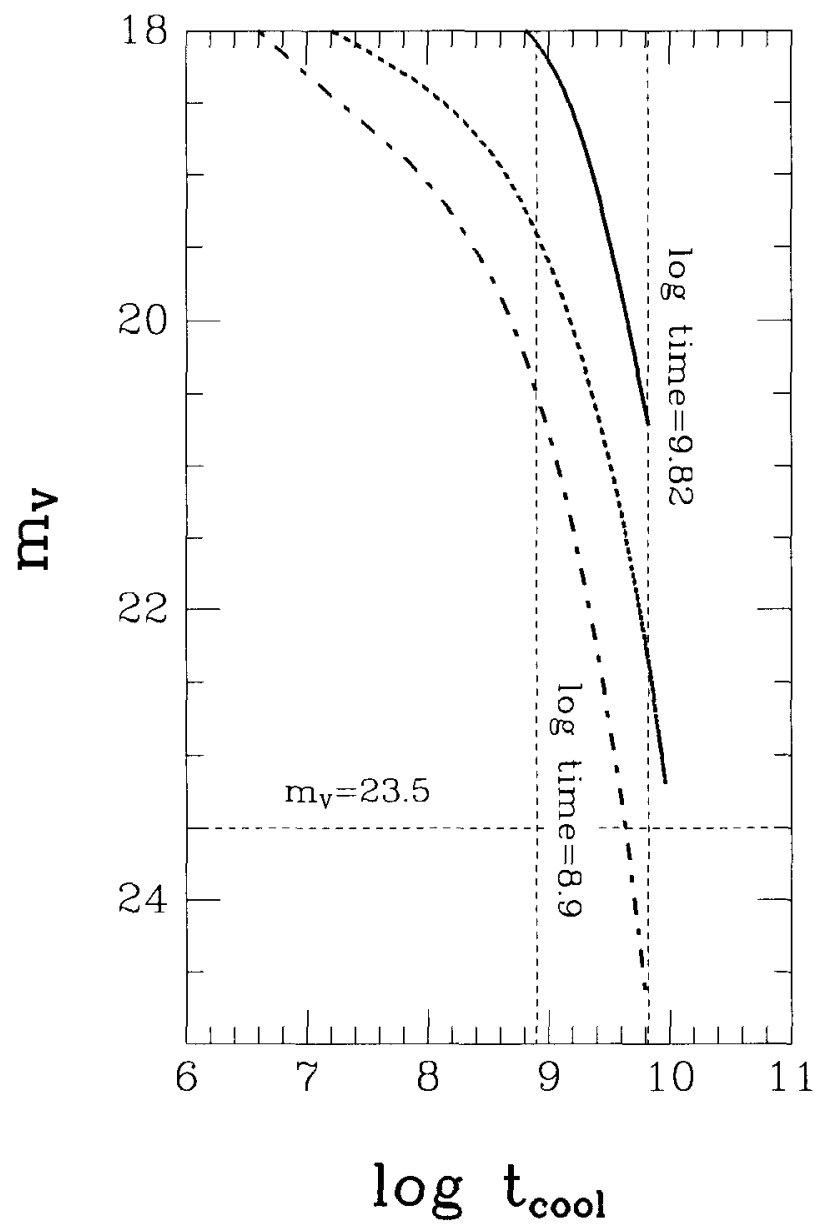

Figure 1. Evolution of the visual magnitude during the cooling phase for the three cases considered. For the wind phase we show three different efficiencies of the wind: $f=0$ (solid line), $f=0.1$ (dashed line) and $\mathrm{f}=0.5$ (dash-dotted line). The thin horizontal dashed line shows the Lundgren et al. (1996) lower bound for visual magnitude. The two thin vertical dashed lines show lower and upper limits for the millisecond pulsar age. 
with an irradiation induced wind gives the right range of visual magnitude. For the upper limit of the pulsar age (spin-down age $\sim 8$ Gyrs) the $m_{V}$ and $T_{\text {eff }}$ are $(22.4,7200 \mathrm{~K})$ for $f=0.1$ and $(24.7,4700 \mathrm{~K})$ for $f=0.5$.

\section{Conclusion}

We propose that for PSR J0751+1807 the irradiation by the pulsar causes irradiation-driven mass loss from secondary, resulting in exhaustion of the hydrogen envelope, and as a consequence hydrogen shell burning stops. The irradiation-driven wind influences also the cooling phase, decreasing the cooling time-scale by a factor 1.7-4 (depending on how efficient this process is). For the PSR J1012+5307 system the irradiative flux may be too small to cause significant mass loss, and hydogren shell burning will be maintained for a long enough time to keep the white dwarf hot.

Acknowledgments. We would like to thank Katrina M. Exter for help in improving the form and text of the paper. This work was supported in part by the State Committee for Scientific Research under grant 2-P03D-005-16, 2-P03D-014-07 and by the Estonian SF grant 2446.

\section{References}

Alberts, F., Savonije, G. J., van den Heuvel, E. P. J., \& Pols, O. R. 1996, Nature, 380,676

Alpar, M. A., Cheng, A. F., Ruderman, M. A., \& Shaham, J. 1982, Nature, 300, 728

Callanan, P. J., Garnavich, P. M., \& Koester, D. 1998, MNRAS, 298, 207

Driebe, T., Schönberner, D., Blöcker, T., \& Herwig, F. 1998, A\&A, 339, 123

Ergma, E., \& Sarna, M. J. 1996, MNRAS, 280, 1000

Ergma, E., Sarna, M. J., \& Antipova, J. 1998, MNRAS, 300, 352

Ergma, E., Sarna, M. J., \& Antipova, J. 1999, MNRAS, in press

Lorimer, D. R., Lyne, A. G., Festin, L., \& Nicastro, L. 1995, Nature, 376, 393

Lundgren, S. C., Zepka, A. F., \& Cordes, J. M. 1995, ApJ, 453, 419

Lundgren, S. C., Cordes, J. M., Foster, R. S., Wolszczan, A., \& Camilo, F. 1996, ApJ, 458, L33

Nicastro, L., Lyne, A. G., Lorimer, D. R., Harrison, P. A., Bailes, M., \& Skidmore, B. D. 1995, MNRAS, 273, L68

Sarna, M. J., Antipova, J., \& Muslimov, A. 1998, ApJ, 499, 407

Sarna, M. J., Ergma, E., \& Antipova, J. 1999, MNRAS, in press

van den Heuvel, E. P. J., \& van Paradijs, J. 1988, Nature, 334, 227

van Kerkwijk, M. H., Bergeron, P., \& Kulkarni, S. R. 1996, ApJ, 467, L41

Webbink, R. F. 1975, MNRAS, 171, 555 